\title{
CURVATURE OF FIELDS OF QUANTUM HILBERT SPACES
}

\author{
LÁSZLó LEMPERT AND RÓBERT SzŐKE*)
}

\begin{abstract}
We show that using the family of adapted Kähler polarizations of the phase space of a compact, simply connected, Riemannian symmetric space of rank-1, the obtained field $H^{\text {corr }}$ of quantum Hilbert spaces produced by geometric quantization including the half-form correction is flat if $M$ is the 3-dimensional sphere and not even projectively flat otherwise.
\end{abstract}

1. Introduction. Suppose an $m$-dimensional compact Riemannian manifold $M$ is the classical configuration space of a mechanical system, the metric corresponding to twice the kinetic energy. To quantize it according to the prescriptions of Kostant and Souriau $[\mathrm{Ko}, \mathrm{So}, \mathrm{Wo}]$, one first passes to phase space $N$, which for the moment is taken $T M \approx T^{*} M$, a symplectic manifold with an exact symplectic form $\omega$, equal to $\sum d q_{j} \wedge d p_{j}$ in the usual local coordinates. The prequantum line bundle is a Hermitian line bundle $E \rightarrow N$ with a connection whose curvature is $-i \omega$. If $M$ is simply connected, the bundle is unique up to a connection preserving Hermitian isomorphism. In any case, one such line bundle is obtained from a real 1-form $a$ on $N$ such that $d a=-\omega$, by letting $E=N \times \mathbb{C} \rightarrow N$ to be the trivial line bundle with $h^{E}(x, \gamma)=|\gamma|^{2}$ the trivial metric on it. If sections are identified with functions $\psi: N \rightarrow \mathbb{C}$, the connection $\nabla^{E}$ is defined by

$$
\nabla_{\zeta}^{E} \psi=\zeta \psi+i a(\zeta) \psi, \quad \zeta \in \operatorname{Vect} N .
$$

A choice of a Kähler structure on $N$ with Kähler form $\omega$ induces on $E$ the structure of a holomorphic line bundle. This gives rise to the quantum Hilbert space $H$, consisting of holomorphic sections of $E$ that are $L^{2}$ with respect to the volume form $\omega^{m} / m !$.

Often one is forced to include in this construction the so called half-form correction. Suppose $\kappa$ is a square root of the canonical bundle $K_{N}$. Then the corrected quantum Hilbert space $H^{\text {corr }}$ consists of the $L^{2}$ holomorphic sections of $E \otimes \kappa$.

When $M$ is a real-analytic Riemannian manifold, there is a natural Kähler polarization on (some subset of) $N$. In [Sz1,GS] the second author and Guillemin-Stenzel construct a canonical complex structure ("adapted complex structure" or "Grauert tube") on a neighborhood $X \subset T M$ of the zero section, in which $\omega$ becomes a Kähler form (see also $[\mathrm{H}-\mathrm{K}]$ ). In good cases $X=N$. One gets examples of this sort when $M$ is a compact normal Riemannian homogeneous space, but there are nonhomogeneous examples as well, see [A,Sz1, Sz2].

2000 Mathematics Subject Classification. 53D50, 53C35, 32L10, 70G45,65.

Key words and phrases. adapted complex structures, geometric quantization, Hilbert fields. * Research partially supported by NSF grant DMS 0700281 and OTKA grants N81203. 
In fact the adapted complex structure is just one member in a natural family of Kähler structures on $N$ [L-Sz2]. To see this it is advantageous to adhere to Souriau's philosophy ([So]) and define the phase space $N$ of a compact Riemannian manifold not as $T M$ or $T^{*} M$ but as the manifold of parametrized geodesics $x: \mathbb{R} \rightarrow M$. Any $t_{0} \in \mathbb{R}$ induces a diffeomorphism $N \ni x \mapsto \dot{x}\left(t_{0}\right) \in T M$, and the pull back of the canonical symplectic form of $T M \approx T^{*} M$ is independent of $t_{0}$; we denote it by $\omega$. We identify $M$ with the submanifold of zero speed geodesics in $N$. Affine reparametrizations $t \mapsto a+b t, a, b \in \mathbb{R}$, act on $N$ and define a right action of the Lie semigroup $\Sigma$ of affine reparametrizations.

Given a complex manifold structure on $\Sigma$, a complex structure on $N$ is called adapted if for every $x \in N$ the orbit map $\Sigma \ni \sigma \mapsto x \sigma \in N$ is holomorphic ([L-Sz2]). An adapted complex structure on $N$ can exist only if the initial compex structure on $\Sigma$ is left invariant. Left invariant complex structures on $\Sigma$ are parametrized by the points of $\mathbb{C} \backslash \mathbb{R}$. For each $s \in \mathbb{C} \backslash \mathbb{R}$ and corresponding left invariant complex structure $I(s)$ on $\Sigma$, if an $I(s)$ adapted complex structure $J(s)$ exists on $N$, then this structure is unique and if $J(i)$ exists, then $J(s)$ also exists for all $s$ in $s \in \mathbb{C} \backslash \mathbb{R}$. The points of the upper half plane (denoted from now on by $S$ ) correspond to $J(s)$ in which $\omega$ is a Kahler form. The original definition of adapted complex structures in [L-Sz1, Sz1] corresponds to the parameter $s=i$.

Now suppose for the compact Riemannian manifold $M$ the adapted complex structure $J(i)$ exists on $N$. With the help of the corresponding family of Kähler structures $J(s)$ on $N$, geometric quantization produces a family $H_{s}$ of quantum Hilbert spaces. Our main concern is how (and when) can one define a natural (projective) isomorphism among these Hilbert spaces.

To deal with this problem, a key idea, following [ADW] and [Hi], is that the collection $\left\{H_{s}: s \in S\right\}$ resembles a holomorphic Hermitian vector bundle, in which one can try to construct a Chern-like canonical connection, and use its parallel transport canonically to identify the different fibers $H_{s}$. To what extent this can be done was explored in [L-Sz3]. The starting point is that the family of adapted complex structures $J(s), s \in S$ on $N$ can all be put together to form a holomorphic fibration $\pi: Y \rightarrow S$; where the fibers $Y_{s}=\pi^{-1} s$ are biholomorphic to $(N, J(s))$. In fact, as a differentiable manifold, $Y=S \times N$, and the projection pr: $Y \rightarrow N$ realizes the biholomorphisms $Y_{s} \rightarrow(N, J(s))$, ([L-Sz2, Theorem 5]).

Armed with this fibration one can perform geometric quantization simultaniously. As we shall see shortly, the object we get is what we call a field of Hilbert spaces. A field of Hilbert spaces is simply a map $p: H \rightarrow S$ of sets with each fiber $H_{s}=p^{-1}(s)$ endowed with the structure of a Hilbert space. When $S$ is a smooth or real analytic manifold, one can introduce the notion of a smooth or analytic structure on $p: H \rightarrow S$, by specifying a set $\Gamma^{\infty}$ resp. $\Gamma^{\omega}$ of sections of $p$, together with operators $\nabla_{\xi}: \Gamma^{\infty} \rightarrow \Gamma^{\infty}$ for all vector fields $\xi$ on $S$. The set $\Gamma^{\infty}$ and the operators $\nabla_{\xi}$ are supposed to satisfy certain axioms (see [L-Sz3, Sect.2]).

Smooth fields of Hilbert spaces are looser structures than Hilbert bundles, but the notion is strong enough to define curvature, (projective) flatness and (local) triviality of the field. In particular a smooth Hilbert field $H \rightarrow S$ is called projectively flat if the curvature operator

$$
R(\xi, \eta)=\nabla_{\xi} \nabla_{\eta}-\nabla_{\eta} \nabla_{\xi}-\nabla_{[\xi, \eta]}: \Gamma^{\infty} \rightarrow \Gamma^{\infty}
$$

is multiplication by a function $r(\xi, \eta): S \rightarrow \mathbb{C}$. Just like with vector bundles, $r$ is in fact a smooth closed 2 -form on $S$, and a simple twisting will reduce projectively flat 
smooth Hilbert fields to flat ones. Flatness and projective flatness are important, because in a flat and analytic field a parallel transport can be introduced that identifies the fibers canonically. Similarly, in projectively flat analytic fields the corresponding parallel transport identifies the projectivized fibers ([L-Sz3, Theorem 2.3.2, Theorem 2.4.2]).

Now back to geometric quantization of a compact Riemannian manifold $M$, assuming the adapted complex structure $J(s)$ exists on the entire manifold $N$ of geodesics. To quantize $(N, J(s))$ simultaneously, construct a Hermitian holomorphic line bundle $E \rightarrow Y$ with curvature $-i \tilde{\omega}:=-i \mathrm{pr}^{*} \omega$. The restriction of $E$ to $Y_{s}$ yields the prequantum line bundle corresponding to $(N, J(s), \omega)$. The restriction of the form $\nu=\tilde{\omega}^{m} / m$ ! to a fiber $Y_{s}$ is a volume form. The spaces of holomorphic $L^{2}$-sections of $E \mid Y_{s}$ form the Hilbert field $H \rightarrow S$. Assuming now that $M$ is simply connected, there is a unique Hermitian holomorphic line bundle $\kappa$ on $Y$, so that $\kappa \otimes \kappa \approx K_{\pi}$ (the relative canonical bundle of $Y$ with $K_{\pi} \mid Y_{s}$ being the canonical bundle of $Y_{s}$ ). The spaces of holomorphic $L^{2}$-sections of $E \otimes \kappa \mid Y_{s}$ form the corrected Hilbert field $H^{\text {corr }} \rightarrow S$.

More generally Hilbert fields naturally arise as direct images of holomorphic vector bundles. Suppose $\pi: Y \rightarrow S$ is a surjective holomorphic submersion of finite dimensional complex manifolds, not necessarily proper. Let $\nu$ be a smooth form on $Y$ that restricts to a volume form on each fiber $Y_{s}=\pi^{-1} s$ and let $\left(E, h^{H}\right) \rightarrow Y$ be a Hermitian holomorphic vector bundle of finite rank. Let $H_{s}$ be the Hilbert space of $L^{2}$ holomorphic sections of $E \mid Y_{s}$. The spaces $H_{s}$ form a Hilbert field $H \rightarrow S$.

Under certain conditions on $Y$ and $E$, the field comes naturally endowed with a smooth structure ([L-Sz3, Sect. 6, 7]). In the problem of geometric quantization by adapted complex structures, these conditions are known to be satisfied in the special case when $M$ is a compact, simply connected, normal Riemannian homogeneous space. In fact, in this case $H^{\text {corr }} \rightarrow S$ turns out to be analytic ([L-Sz3, Theorem 11.1.1]).

Our main result is the following:

Theorem 1.1. Let $M$ be a compact, simply connected, Riemannian symmetric space of rank-1. Then the corresponding field $H^{\text {corr }}$ of quantum Hilbert spaces is flat if $M$ is the 3-dimensional sphere and not even projectively flat otherwise.

We prove this result in Sect.5. It shows quantization is unique for the 3 -sphere and in the rest of the cases quantization does depend on the choice of the Kähler polarization.

Flatness also implies $H^{\text {corr }} \rightarrow S$ is a genuine Hilbert bundle (trivial in this case), something that is not known to be true for the other rank-1 symmetric spaces.

The situation for the higher rank symmetric spaces is more complicated and will be treated in a separate publication [L-Sz4].

\section{Curvature calculations.}

Consider a simply connected, compact, Riemannian symmetric space $\left(M^{m}, g\right)$ and $H^{\text {corr }} \rightarrow S$ the corresponding field of quantum Hilbert spaces. Let $U$ denote the identity component of the isometry group of $M$ and $K \subset U$ the isotropy group of a fixed $o \in M$. Let $\mathfrak{u}$ and $\mathfrak{k}$ be the Lie algebras of $U$ and $K$ and let $\mathfrak{p}_{*} \subset \mathfrak{u}$ be the orthogonal complement of $\mathfrak{k}$.

$U$ acts on $(N, J(i))$ by biholomorphisms and this action induces a representation $\hat{\pi}$ on $\mathcal{O}(N, J(i))$, by the formula $a v=\left(a^{-1}\right)^{*} v$ (pull back by $a^{-1}$, where $a \in U$, 
$v \in \mathcal{O}(N, J(i))$. The same formula defines a unitary representation $\pi$ on $L^{2}(M)$. The restrictions $\left.V_{\chi}\right|_{M}$ of the isotypical subspaces of $\hat{\pi}$ are precisely the isotypical subspaces of $\pi$ and the latter are well known to be finite dimensional. Since $M$ is a maximal dimensional, totally real submanifold in $N$, we get that $V_{\chi}$ are also finite dimensional. The isotypical subspaces of $\pi$ are parametrized by the irreducible spherical (w.r.t. $K$ ) representations of $U$ ([He2, Theorem 4.3]). In fact the restrictions of $\hat{\pi}$ to the isotypical subspaces $V_{\chi}$ (or equivalently the restrictions of $\pi$ to $\left.\left.V_{\chi}\right|_{M}\right)$ are precisely these spherical representations.

Flatness of the field $H^{\text {corr }} \rightarrow S$ can be understood in terms of certain operators $P_{\chi}(s)$ on $V_{\chi}$. Namely $H^{\text {corr }} \rightarrow S$ is flat (resp. projectively flat) if and only if $P_{\chi}(s)$ are of the form $P_{\chi}(s)=p_{\chi}(s) I d_{\chi}$ and $\bar{\partial} \partial \log p_{\chi}(s)=0$ for all $\chi\left(\right.$ resp. $\bar{\partial} \partial \log p_{\chi}(s)$ is independent of $\chi$ ), see [L-Sz3, Theorem 9.2.1]. According to [L-Sz3, Lemma 11.2.1] and [L-Sz3, Sect. 12.1],

$$
p_{\chi}(s)=\frac{c_{\chi}}{(\operatorname{Im} s)^{m / 2}} \int_{\mathfrak{p}_{*}} \int_{K} e^{-\frac{|\zeta|^{2}}{\operatorname{Im} s}} \chi(k \exp (-2 i \zeta)) \sqrt{\eta(\zeta)} d k d \zeta
$$

where $c_{\chi}$ is independent of $s, d k$ is normalized Haar measure on $K, d \zeta$ translation invariant Lebesgue measure on $\mathfrak{p}_{*}$, and

$$
\eta(\zeta):=\operatorname{det}\left(\left.\frac{\sin 2 \mathrm{ad} \zeta}{\operatorname{ad} \zeta}\right|_{\mathbb{C} \otimes \mathfrak{p}_{*}}\right) .
$$

The function $f_{\chi}(g)=\int_{K} \chi\left(k^{-1}\right) d k$, occuring in (2-1), is known as spherical function, corresponding to the character $\chi$, see [He2, IV., Theorem 4.2]. This function has a holomorphic extension to the complexified group $U_{\mathbb{C}}$ that we also denote by $f_{\chi}$.

Proposition 2.1. The function $f_{\chi} \circ \exp$ is $A d_{K}$ invariant on the Lie algebra $\mathfrak{u}_{\mathbb{C}}$ of $U_{\mathbb{C}}$.

Proof. For any $k, k_{0} \in K, \zeta \in \mathfrak{u}_{\mathbb{C}}$

$$
\chi\left(k \exp \left(-\operatorname{Ad}\left(k_{0}\right) \zeta\right)\right)=\chi\left(k k_{0} \exp (-\zeta) k_{0}^{-1}\right)=\chi\left(k_{0}^{-1} k k_{0} \exp (-\zeta)\right) .
$$

Thus

$$
f_{\chi}\left(\exp \left(\operatorname{Ad}\left(k_{0}\right) \zeta\right)\right)=\int_{K} \chi\left(k_{0}^{-1} k k_{0} \exp (-\zeta)\right) d k=f_{\chi}(\exp (\zeta))
$$

Proposition 2.2. Let $F \in \mathcal{O}(\mathbb{C})$ be an even function and $v \in \mathfrak{p}_{*}$. Then $F(\operatorname{ad}(v))$ (defined by its power series) maps $\mathbb{C} \otimes \mathfrak{p}_{*}$ into itself and $\operatorname{det}\left(\left.F(\operatorname{ad}(v))\right|_{\mathbb{C} \otimes \mathfrak{p}_{*}}\right)$ is an $A d_{K}$ invariant function.

Proof. For every $k$ in $K, \operatorname{Ad}(k)$ is in $\operatorname{Aut}(\mathfrak{u})$. Thus for every $v \in \mathfrak{u}, l=0,1, \ldots$

$$
(\operatorname{ad}(\operatorname{Ad}(k) v))^{l}=\operatorname{Ad}(k) \circ(\operatorname{ad}(v))^{l} \circ \operatorname{Ad}(k)^{-1} .
$$

Hence

$$
F(\operatorname{ad}(\operatorname{Ad}(k) v))=\operatorname{Ad}(k) \circ F(\operatorname{ad}(v)) \circ \operatorname{Ad}(k)^{-1} .
$$


Since $\mathfrak{p}_{*}$ is both $\operatorname{Ad}(k)$ and $(\operatorname{ad}(v))^{2 l}$ invariant $(l=0,1 \ldots)$, the statement follows.

From now on we shall assume that $M$ is a rank-1 symmetric space.

Let $H_{0} \in \mathfrak{p}_{*}$ with $\left\|H_{0}\right\|=1$. Then $\mathfrak{a}_{*}=\mathbb{R} H_{0}$ is maximal Abelian in $\mathfrak{p}_{*}$ (resp. $\mathfrak{a}:=i \mathfrak{a}_{*}$ in $\left.\mathfrak{p}:=i \mathfrak{p}_{*}\right)$. Let $\Sigma$ be the set of restricted roots corresponding to $\left(\mathfrak{g}_{0}:=\right.$ $\mathfrak{k}+\mathfrak{p}, \mathfrak{a})$. Let $\mathfrak{a}^{+}:=\left\{i r H_{0} \mid r>0\right\}$ be the Weyl chamber and $\Sigma^{+}$the set of positive restricted roots. Then $\Sigma^{+}=\{\beta, \beta / 2\}$ with an apropriate $\beta$ in the dual of $\mathfrak{p}$ with $B:=\beta\left(i H_{0}\right)>0$. The corresponding multiplicities are $m_{\beta}$ and $m_{\beta / 2}$, where our convention is that the latter is zero when $\Sigma$ is reduced (i.e. when $M$ is a sphere with the round metric).

Let $\mathbb{Z}_{+}=\{0,1,2, \ldots\}$. According to Helgason's theorem ([He2, Theorem 4.1,(ii), p.535 and Sect.3, p.542]), the set of linear functionals $\left\{\mu=n \beta: n \in \mathbb{Z}_{+}\right\}$is precisely the set of the highest weights of all irreducible spherical representations of $U$ (w.r.t. $K)$ restricted to $\mathfrak{a}$. Now in light of what was said at the beginning of Sect.2 about the relationship of $V_{\chi}$ and the irreducible spherical representations of $U$, we can conclude that the isotypical subspaces $V_{\chi}$ of $\hat{\pi}$ are parametrized by the elements $n_{\chi}$ of $\mathbb{Z}_{+}$. Let

$$
a_{\chi}=\frac{1}{2} m_{\beta / 2}+m_{\beta}+n_{\chi}, \quad b_{\chi}=-n_{\chi}, \quad c_{\chi}=\frac{m_{\beta / 2}+m_{\beta}+1}{2}=\frac{m}{2},
$$

and denote by $F_{\chi}$ the Gauss hypergeometric function, corresponding to these parameters

$$
F_{\chi}(x)=F\left(a_{\chi}, b_{\chi}, c_{\chi}, x\right) .
$$

(see Sect.5 for more on hypergeometric functions).

Let $S_{\mathfrak{p}_{*}}^{m-1}$ be the unit sphere in the euclidean space $\mathfrak{p}_{*}$.

Theorem 2.3. Let $M^{m}$ be a compact, simply connected, rank-1 symmetric space. Then

$$
p_{\chi}(s)=\frac{c_{\chi} \operatorname{Vol}\left(S_{\mathfrak{p}_{*}}^{m-1}\right) 2^{\frac{m}{2}}}{B^{m}(\operatorname{Im} s)^{\frac{m}{2}}} \int_{0}^{\infty} e^{-\frac{t^{2}}{B^{2} \operatorname{Im} s}} F_{\chi}\left(-\operatorname{sh}^{2}(t)\right) t^{\frac{m-1}{2}}(\operatorname{sh}(t))^{\frac{m-1}{2}}(\operatorname{ch}(t))^{\frac{m_{\beta}}{2}} d t .
$$

Proof. Suppose $f \in L^{1}\left(\mathfrak{p}_{*}\right)$ depends only on $\|\zeta\|$. Using polar coordinates we obtain

$$
\int_{\mathfrak{p}_{*}} f(\zeta) d \zeta=\operatorname{Vol}\left(S_{\mathfrak{p}_{*}}^{m-1}\right) \int_{0}^{\infty} f\left(r H_{0}\right) r^{m-1} d r
$$

According to [He2, formula (25), p.543], the spherical function $f_{\chi}$ can be expressed as

$$
f_{\chi}\left(\exp \left(2 i r H_{0}\right)\right)=F_{\chi}\left(-\operatorname{sh}^{2}\left(\beta\left(i r H_{0}\right)\right)\right)=F_{\chi}\left(-\operatorname{sh}^{2}(r B)\right) .
$$

Prop.2.2 applied to $F(z)=\frac{\sin 2 z}{z}$ shows that $\eta$ is $\operatorname{Ad}_{K}$ invariant. Since the rank is $1, \mathrm{Ad}_{K}$ acts transitively on each sphere with center the origin in $\mathfrak{p}_{*}$. In light of Prop.2.1, (2-5) applies to the integrand in (2-1) and we get

$$
p_{\chi}(s)=\frac{c_{\chi} \operatorname{Vol}\left(S_{\mathfrak{p}_{*}}^{m-1}\right)}{(\operatorname{Im} s)^{\frac{m}{2}}} \int_{0}^{\infty} e^{-\frac{r^{2}}{\operatorname{Im} s}} F_{\chi}\left(-\operatorname{sh}^{2}(r B)\right) r^{m-1} \sqrt{\eta}\left(r H_{0}\right) d r .
$$


For $H \in \mathfrak{a}_{*}, \operatorname{ad} H^{2}: \mathfrak{p}_{*} \rightarrow \mathfrak{p}_{*}$ has eigenvalues 0 with multiplicity $1, \beta(H)^{2}$ with multiplicity $m_{\beta}$ and $(\beta(H) / 2)^{2}$ with multiplicity $m_{\beta / 2}([\operatorname{He} 1$, Lemma 2.9, p288]). Thus

$$
\eta(H)=2\left(\frac{\sin (2 \beta(H))}{\beta(H)}\right)^{m_{\beta}}\left(\frac{\sin (\beta(H))}{\beta(H) / 2}\right)^{m_{\beta / 2}} .
$$

Now for $H=r H_{0}$ we have $\beta\left(r H_{0}\right)=-i r B$. Hence $(2-8)$ yields

$$
\eta\left(r H_{0}\right)=\frac{2^{m}}{(r B)^{m-1}}(\operatorname{sh}(r B))^{m-1}(\operatorname{ch}(r B))^{m_{\beta}} .
$$

Substituting this into (2-7) and changing the variable $r$ in the integral to $t=r B$ we finally get formula (2-4).

From (2-4) we see that $p_{\chi}(s)$ depends only on $\tau=B^{2} \operatorname{Im} s$. In light of our earlier characterization of the (projective) flatness of $H^{\text {corr }} \rightarrow S$ in term of $\bar{\partial} \partial \log p_{\chi}$ we obtain:

Corollary 2.4. Let $M$ be a compact, simply connected rank-1 symmetric space. For $\tau>0$ let

$$
q_{\chi}(\tau):=\int_{0}^{\infty} e^{-\frac{t^{2}}{\tau}} F_{\chi}\left(-\operatorname{sh}^{2}(t)\right) t^{\frac{m-1}{2}}(\operatorname{sh}(t))^{\frac{m-1}{2}}(\operatorname{ch}(t))^{\frac{m_{\beta}}{2}} d t
$$

Then the field of quantum Hilbert spaces $H^{\text {corr }} \rightarrow S$ is

$$
\begin{array}{r}
\text { flat iff }\left(\log q_{\chi}(\tau)\right)^{\prime \prime} \equiv 0 \text { for every } \chi \text {, } \\
\text { projectively flat iff }\left(\log q_{\chi}(\tau)\right)^{\prime \prime} \text { does not depend on } \chi \text {. }
\end{array}
$$

The integral in (2-9) can be explicitely calculated only in very special cases. To be able to decide whether (2-10) holds, we shall use asymptotic methods to investigate the behavior of $q_{\chi}(\tau)$ as $\tau \rightarrow 0$ and $\tau \rightarrow \infty$. As we will see in Sect.5, the function $F_{\chi}(x)$ is a polynomial of degree $n_{\chi}$. This motivates our investigations in the next sections.

\section{3. $Q_{P}$ functions and central polynomial sequences.}

Let $P(x)=c_{n} x^{n}+\cdots+c_{1} x+c_{0}$ be a polynomial $\left(c_{j} \in \mathbb{C}\right), \tau>0$ and $\mu, \kappa, \nu \in \mathbb{C}$, with $\operatorname{Re}(\mu+\kappa)>-1$. Define the corresponding $Q_{P}$ function by the formula

$$
Q_{P}(\tau):=\int_{0}^{\infty} e^{-\frac{t^{2}}{\tau}} P\left(-\operatorname{sh}^{2} t\right) t^{\mu}(\operatorname{sh} t)^{\kappa}(\operatorname{ch} t)^{\nu} d t
$$

The integral converges absolutely and $Q_{P}$ depends holomorphically on the parameters $\mu, \kappa, \nu$. The function

$$
f_{P}(t):=P\left(-\operatorname{sh}^{2} t\right)\left(\frac{\operatorname{sh} t}{t}\right)^{\kappa}(\operatorname{ch}(t))^{\nu}
$$


is even and extends holomorphically to a neighborhood of the real line. Let $r:=$ $\kappa+\mu+1$. Then

$$
Q_{P}(\tau)=\int_{0}^{\infty} e^{-\frac{t^{2}}{\tau}} t^{r-1} f_{P}(t) d t
$$

Applying Watson's lemma [W] to this integral we get

$$
Q_{P}(\tau)=\frac{\tau^{\frac{r}{2}}}{2}\left(\Gamma\left(\frac{r}{2}\right) f_{P}(0)+\Gamma\left(\frac{r}{2}+1\right) \frac{f_{P}^{\prime \prime}(0)}{2} \tau+o(\tau)\right), \quad \tau \rightarrow 0,
$$

where $\Gamma$ denotes the usual gamma function. Now $f_{P}(0)=c_{0}$ and a straightforward calculation shows

$$
\frac{f_{P}^{\prime \prime}(0)}{2}=-c_{1}+\frac{\kappa}{6}+\frac{\nu}{2}
$$

and we get:

\section{Proposition 3.1.}

$$
Q_{P}(\tau)=\frac{\tau^{\frac{r}{2}}}{2}\left(\Gamma\left(\frac{r}{2}\right) c_{0}+\Gamma\left(\frac{r}{2}+1\right)\left(-c_{1}+\frac{\kappa}{6}+\frac{\nu}{2}\right) \tau+o(\tau)\right), \quad \tau \rightarrow 0
$$

The next definition is motivated by Corollary 2.4 .

Definition 3.2. Let $\left\{P_{n}(x)=c_{n, n} x^{n}+\cdots+c_{n, 1} x+1\right\}_{n=0}^{\infty}, c_{n, n} \neq 0\left(c_{n, j} \in \mathbb{C}\right)$ be a sequence of polynomials. The sequence is called central (w.r.t. the parameters $\mu, \kappa, \nu)$ if the function $\left(\log Q_{P_{n}}\right)^{\prime \prime}$ does not depend on $n$.

Proposition 3.3. Suppose $\left\{P_{n}\right\}_{n=0}^{\infty}$ is a central sequence of polynomials. Then

$$
Q_{P_{n}}(\tau)=e^{-\frac{r}{2} c_{n, 1} \tau} Q_{1}(\tau), \quad n \geq 1, \quad \text { where } r=\mu+\kappa+1
$$

Proof. From our assumption

$$
\left(\log Q_{P_{n}}-\log Q_{P_{0}}\right)^{\prime \prime} \equiv 0,
$$

for every $n$. Hence there exist constants $\alpha_{n}$ and $\beta_{n}$ such that

$$
Q_{P_{n}}(\tau)=\beta_{n} e^{\alpha_{n} \tau} Q_{P_{0}}(\tau)
$$

Substituting this into the asymptotic formula in Prop.3.1 we get

$$
\begin{aligned}
\beta_{n}\left(1+\alpha_{n} \tau+o(\tau)\right) & \frac{\tau^{\frac{r}{2}}}{2}\left(\Gamma\left(\frac{r}{2}\right)+\Gamma\left(\frac{r}{2}+1\right)\left(\frac{\kappa}{6}+\frac{\nu}{2}\right) \tau+o(\tau)\right)= \\
& =\frac{\tau^{\frac{r}{2}}}{2}\left(\Gamma\left(\frac{r}{2}\right)+\Gamma\left(\frac{r}{2}+1\right)\left(-c_{n, 1}+\frac{\kappa}{6}+\frac{\nu}{2}\right) \tau+o(\tau)\right) .
\end{aligned}
$$

Now dividing by $\frac{\tau^{\frac{r}{2}}}{2}$ and comparing the constant term and the coefficient of $\tau$ on both sides of (3-6) we get

$$
\beta_{n}=1
$$

and

$$
\beta_{n} \alpha_{n} \Gamma\left(\frac{r}{2}\right)+\Gamma\left(\frac{r}{2}+1\right) \beta_{n}\left(\frac{\kappa}{6}+\frac{\nu}{2}\right)=\Gamma\left(\frac{r}{2}+1\right)\left(-c_{n, 1}+\frac{\kappa}{6}+\frac{\nu}{2}\right) .
$$

Hence $\alpha_{n}=-r c_{n, 1} / 2$. 


\section{Asymptotics at infinity.}

Proposition 4.1. Let $a \geq 0, \lambda>0, \mu>-1, \tau>0$. Then

$$
I(\tau):=\int_{a}^{\infty} e^{-t^{2} / \tau} t^{\mu} e^{\lambda t} d t=\frac{\lambda^{\mu} \sqrt{\pi}}{2^{\mu}} \tau^{\mu+1 / 2} e^{\lambda^{2} \tau / 4}(1+o(1)), \quad \tau \longrightarrow \infty .
$$

Proof. We can rewrite $I(\tau)$ as follows:

$$
I(\tau)=e^{\lambda^{2} \tau / 4} \int_{a}^{\infty} e^{-\left(\frac{t}{\sqrt{\tau}}-\frac{\lambda \sqrt{\tau}}{2}\right)^{2}} t^{\mu} d t
$$

After the substitution $x=\frac{t}{\sqrt{\tau}}-\frac{\lambda \sqrt{\tau}}{2}$, we get

$$
I(\tau)=\tau^{\mu+1 / 2} e^{\lambda^{2} \tau / 4} \int_{\frac{a}{\sqrt{\tau}}-\frac{\lambda \sqrt{\tau}}{2}}^{+\infty} e^{-x^{2}}\left(\frac{x}{\sqrt{\tau}}+\frac{\lambda}{2}\right)^{\mu} d x=: \tau^{\mu+1 / 2} e^{\lambda^{2} \tau / 4} I_{2}(\tau) .
$$

Lebesgue's dominated convergence theorem applied to $I_{2}(\tau)$ yields:

$$
I_{2}(\tau) \longrightarrow\left(\frac{\lambda}{2}\right)^{\mu} \int_{-\infty}^{+\infty} e^{-x^{2}} d x=\left(\frac{\lambda}{2}\right)^{\mu} \sqrt{\pi}, \quad \tau \rightarrow \infty
$$

finishing the proof.

Proposition 4.2. Let $\nu>0, \kappa>0, \mu+\kappa>-1 \tau>0$. Then

$$
\int_{0}^{\infty} e^{-\frac{t^{2}}{\tau}} t^{\mu}(\operatorname{sh} t)^{\kappa}(\operatorname{ch} t)^{\nu} d t=\frac{\sqrt{\pi}(\nu+\kappa)^{\mu}}{2^{\mu+\nu+\kappa}} \tau^{\mu+\frac{1}{2}} e^{\frac{(\kappa+\nu)^{2}}{4} \tau}(1+o(1)), \quad \tau \rightarrow \infty .
$$

Proof. Let $a>0$ be an arbitrary fixed constant. Then

$$
\int_{0}^{a} e^{-\frac{t^{2}}{\tau}} t^{\mu}(\operatorname{sh} t)^{\kappa}(\operatorname{ch} t)^{\nu} d t=O(1)=\tau^{\mu+\frac{1}{2}} e^{\frac{(\kappa+\nu)^{2}}{4} \tau} o(1), \quad \tau \rightarrow \infty .
$$

Let $h(x)=(1-x)^{\kappa}(1+x)^{\nu}$. Then $h \in C^{\infty}(-1,1), h(0)=1$. Hence $h(x)=1+x g(x)$, where $g \in C^{\infty}(-1,1)$. Therefore

$$
(\operatorname{sh} t)^{\kappa}(\operatorname{ch} t)^{\nu}=\frac{e^{(\kappa+\nu) t}}{2^{\kappa+\nu}}\left(1-e^{-2 t}\right)^{\kappa}\left(1+e^{-2 t}\right)^{\nu}=\frac{e^{(\kappa+\nu) t}}{2^{\kappa+\nu}}\left(1+e^{-2 t} g\left(e^{-2 t}\right)\right)
$$

Thus

$$
\begin{aligned}
& \int_{a}^{\infty} e^{-\frac{t^{2}}{\tau}} t^{\mu}(\operatorname{sh} t)^{\kappa}(\operatorname{ch} t)^{\nu} d t= \\
& =\frac{1}{2^{\kappa+\nu}} \int_{a}^{\infty} e^{-\frac{t^{2}}{\tau}} t^{\mu} e^{(\kappa+\nu) t} d t+\frac{1}{2^{\kappa+\nu}} \int_{a}^{\infty} e^{-\frac{t^{2}}{\tau}} t^{\mu} e^{(\kappa+\nu-2) t} g\left(e^{-2 t}\right) d t=I_{1}+I_{2} .
\end{aligned}
$$


Now by Proposition 4.1

$$
I_{1}=\frac{\sqrt{\pi}(\nu+\kappa)^{\mu}}{2^{\mu+\nu+\kappa}} \tau^{\mu+\frac{1}{2}} e^{\frac{(\kappa+\nu)^{2}}{4}} \tau(1+o(1)) .
$$

The function $g\left(e^{-2 t}\right)$ is bounded on $[a, \infty)$, thus for an appropriate constant $A$ we have

$$
\left|I_{2}\right| \leq A \int_{0}^{\infty} e^{-\frac{t^{2}}{\tau}} t^{\mu} e^{(\kappa+\nu-2) t} d t=: A I_{3}
$$

If $\kappa+\nu-2>0$, by Proposition 4.1

$$
I_{3}=\tau^{\mu+\frac{1}{2}} e^{\frac{(\kappa+\nu-2)^{2}}{4} \tau} O(1)=\tau^{\mu+\frac{1}{2}} e^{\frac{(\kappa+\nu)^{2}}{4} \tau} o(1) .
$$

If $\kappa+\nu-2 \leq 0$, after the substitution $t=\sqrt{\tau x}$ we obtain

$$
I_{3} \leq \int_{0}^{\infty} e^{-\frac{t^{2}}{\tau}} t^{\mu} d t=\frac{\tau^{\frac{\mu+1}{2}}}{2} \int_{0}^{\infty} e^{-x} x^{\frac{\mu-1}{2}} d x=\frac{\tau^{\frac{\mu+1}{2}}}{2} \Gamma\left(\frac{\mu+1}{2}\right)
$$

Thus in both cases

$$
I_{2}=\tau^{\mu+\frac{1}{2}} e^{\frac{(\kappa+\nu)^{2}}{4} \tau} o(1)
$$

Then (4-1), (4-2), (4-3) and (4-4) together prove our claim.

Proposition 4.3. Let $\nu>0, \kappa>0, \mu+\kappa>-1$ and $P(t)=c_{n} t^{n}+\cdots+c_{0}$ be $a$ polynomial. Let $Q_{P}$ be the corresponding $Q$ function (see (3-1)). Then

$$
Q_{P}(\tau)=(-1)^{n} c_{n} \frac{\sqrt{\pi}(\nu+\kappa+2 n)^{\mu}}{2^{\mu+\nu+\kappa+2 n}} \tau^{\mu+\frac{1}{2}} e^{\frac{(\kappa+\nu+2 n)^{2}}{4} \tau}(1+o(1)), \quad \tau \rightarrow \infty
$$

Proof. It is enough to show the statement for the special case $P(t)=t^{k}$. Then

$$
Q_{P}(\tau)=\int_{0}^{\infty} e^{-\frac{t^{2}}{\tau}}\left(-\operatorname{sh}^{2} t\right)^{k} t^{\mu}(\operatorname{sh} t)^{\kappa}(\operatorname{ch} t)^{\nu} d t=(-1)^{k} \int_{0}^{\infty} e^{-\frac{t^{2}}{\tau}} t^{\mu}(\operatorname{sh} t)^{\kappa+2 k}(\operatorname{ch} t)^{\nu} d t
$$

and Proposition 4.2 proves our claim.

Theorem 4.4. Let $\nu>0, \kappa>0, \mu+\kappa>-1$. Suppose $\left\{P_{n}\right\}_{n=0}^{\infty}$ is a central sequence of polynomials. Then for all $n$

$$
\begin{gathered}
Q_{P_{n}}(\tau)=e^{n(\nu+\kappa+n) \tau} Q_{1}(\tau), \\
c_{n, 1}=-\frac{2 n(\nu+\kappa+n)}{\mu+\kappa+1}, \quad c_{n, n}=(-1)^{n} 4^{n}\left(\frac{\nu+\kappa}{\nu+\kappa+2 n}\right)^{\mu} .
\end{gathered}
$$


Proof. Centrality implies (see (3-4)) that $Q_{P_{n}}(\tau)=A_{n} e^{B_{n} \tau} Q_{1}(\tau)$ for appropriate constants $A_{n}, B_{n}$. Substituting into this the asymptotics of Proposition 4.3 and comparing the leading terms on both sides we get

$$
c_{n, n}(-1)^{n} \frac{(\nu+\kappa+2 n)^{\mu}}{4^{n}} e^{\frac{(\nu+\kappa+2 n)^{2}}{4} \tau}=A_{n} e^{B_{n} \tau}(\nu+\kappa)^{\mu} e^{\frac{(\nu+\kappa)^{2}}{4} \tau} .
$$

Therefore

$$
B_{n}=\frac{(\nu+\kappa+2 n)^{2}-(\nu+\kappa)^{2}}{4}=n(\nu+\kappa+n)
$$

and

$$
A_{n}=c_{n, n}(-1)^{n}\left(\frac{\nu+\kappa+2 n}{\nu+\kappa}\right)^{\mu} \frac{1}{4^{n}} .
$$

Now comparing these expressions of $A_{n}, B_{n}$ with (3-4) we get (4-5) and (4-6).

Taking $n=1$ in (4-6) we get two different expressions for the same coefficient and thus we obtain the following.

Corollary 4.5. Let $\nu, \kappa>0, \mu+\kappa>-1$. Suppose there exists a central sequence of polynomials corresponding to these parameters. Then

$$
\frac{\nu+\kappa+1}{\mu+\kappa+1}=2\left(\frac{\nu+\kappa}{\nu+\kappa+2}\right)^{\mu}
$$

\section{Hypergeometric polynomials and the proof of Theorem 1.1.}

Recall that Gauss' hypergeometric functions are given by

$F(a, b, c, z):=1+\frac{a b}{c} z+\cdots+\frac{a(a+1) \ldots(a+k-1) b(b+1) \ldots(b+k-1)}{k ! c(c+1) \ldots(c+k-1)} z^{k}+\ldots$

where $a, b, c \in \mathbb{C}, c \notin \mathbb{Z}_{-}=\{0,-1,-2, \ldots\}$. The series converges at least in the unit disk. If $n \in \mathbb{Z}_{+}=\{0,1,2, \ldots\}, b=-n, A \in \mathbb{C} \backslash \mathbb{Z}_{-}$, and $a=A+n$, then $F$ is a polynomial (in $z$ ) of degree $n$. Now assume $A, c \in \mathbb{R} \backslash \mathbb{Z}_{-}, n \in \mathbb{Z}_{+}$and consider this sequence of polynomials

$$
P_{n}(x):=F(A+n,-n, c, x)=\sum_{j=0}^{n} c_{n, j} x^{j} .
$$

Proposition 5.1. Suppose the polynomial sequence $P_{n}(x)$ is central w.r.t. some choice of the parameters $\nu, \kappa, \mu$ with $\nu>0, \kappa>0, \mu+\kappa>-1$. Then $A>0, c>0$,

$$
\frac{\Gamma(A+2 n)}{\Gamma(A+n)} \frac{\Gamma(c)}{\Gamma(c+n)}=4^{n}\left(\frac{A}{A+2 n}\right)^{\mu},
$$

and

$$
\kappa=2 c-\mu-1, \quad \nu=A-\kappa .
$$


Proof. Assume $\left\{P_{n}\right\}_{0}^{\infty}$ is central. Then (5-1) and (4-6) yield

$$
c_{n, 1}=\frac{(A+n)(-n)}{c}=-\frac{2 n(\nu+\kappa+n)}{\mu+\kappa+1},
$$

for every $n$, which implies

$$
c=(\mu+\kappa+1) / 2, \quad A=\nu+\kappa,
$$

proving (5-3) and $A, c>0$. From (5-1) we also get

$$
c_{n, n}=(-1)^{n} \frac{\Gamma(A+2 n)}{\Gamma(A+n)} \frac{\Gamma(c)}{\Gamma(c+n)},
$$

which together with formula (4-9) and (5-4) proves (5-2).

Proof of Theorem 1.1. Suppose the field $H^{\text {corr }}$ of quantum Hilbert spaces corresponding to $M$ is projectively flat. As in Section 2, with $M$ we associate its system of restricted roots $\Sigma$. We denote the longer positive restricted root $\beta$, and the multiplicities of $\beta, \beta / 2$ by $m_{\beta}, m_{\beta / 2}$ (with the understanding that this latter is 0 if $\Sigma$ is reduced, so that $\beta / 2$ is not a root, i.e. when $M$ is a sphere). Then $m=m_{\beta}+m_{\beta / 2}+1$. Set

$$
A=m_{\beta}+\frac{m_{\beta / 2}}{2}, \quad c=\frac{m}{2},
$$

and $P_{n}(x)=F(A+n,-n, c, x), n=1,2, \ldots, P_{0}(x)=1$ the corresponding sequence of hypergeometric polynomials. Let also $\mu=\kappa=(m-1) / 2, \nu=m_{\beta} / 2$. According to Corollary 2.4 and Definition 3.2, projective flatness implies that $P_{n}$ is central with respect to $\kappa, \mu, \nu$.

We now apply Proposition 5.1. Choose $n=2 A$, so that $A /(A+2 n)=1 / 5$. The left hand side of $(5-2)$ is rational, hence $\mu=(m-1) / 2$ on the right must be an integer and so $m$ must be odd. Then it follows from the classification of compact rank-1 symmetric spaces (see [He2, Ch.I, Sect.4.2]), that $M$ must be an odd dimensional sphere and so $\Sigma$ is reduced. Thus $m_{\beta / 2}=0$, and $A=m_{\beta}=m-1$. Substitute $n=1$ into (5-2):

$$
2=\left(\frac{m+1}{m-1}\right)^{\frac{m-1}{2}}=\left(1+\frac{2}{m-1}\right)^{\frac{m-1}{2}} .
$$

Clearly $m=3$ solves this equation, and there is no other solution, because $(1+1 / x)^{x}$ is strictly increasing for $x>0$. Thus $M$ must be the 3 dimensional sphere: for no other compact simply connected symmetric space of rank 1 can the Hilbert field $H^{\text {corr }}$ be projectively flat.

On the other hand, when $M$ is $S^{3}$, or more generally a compact Lie group with biinvariant metric, the associated Hilbert field is outright flat, see [L-Sz, Theorem 11.3.1]. 


\section{REFERENCES}

[A] R. M. Aguilar, Symplectic reduction and the homogeneous complex Monge-Ampére equation, Ann. Glob. Anal. Geom. 19 (2001), 327-353.

[ADW] S. Axelrod, S. Della Pietra, E. Witten, Geometric quantization of Chern-Simons gauge theory, J. Diff. Geo. 33 (1991), 787-902.

[GS] V. Guillemin, M. Stenzel, Grauert tubes and the homogeneous Monge-Ampère equation, J. Diff. Geom. 34 (1991), 561-570.

[H-K] B. C. Hall, W. D. Kirwin, Adapted complex structures and the geodesic flow, Math. Ann. 350 (2011), no. 2, 455-474.

[He1] S. Helgason, Differential geometry, Lie groups, and symmetric spaces, Amer. Math. Soc., Providence, 2001.

[He2] - Groups and geometric analysis, integral geometry, invariant differential operators and spherical functions, Amer. Math. Soc., Providence, 2002.

[Hi] N. Hitchin, Flat connections and geometric quantization, Comm. Math. Phys. 131 (1990), 347-380.

[Ko] B. Kostant, Quantization and unitary representations I., Lectures in modern analysis and applications III, Lecture Notes in Math., vol. 170, Springer, Berlin, 1970, pp. 87-208.

[L-Sz1] L. Lempert and R. Szőke, Global solutions of the homogeneous complex Monge-Ampére equation and complex structures on the tangent bundle of Riemannian manifolds, Math. Ann 290 (1991), 689-712.

[L-Sz2] _ A new look at adapted complex structures, doi:10.1112/blms/bdr097, Bull. of LMS (2011).

[L-Sz3] - Uniqueness in geometric quantization, arXiv:1004.4863.

[L-Sz4] - Root systems and curvature of fields of quantum Hilbert spaces, work in progress.

[So] J.-M. Souriau, Structure des systèmes dynamiques, Dunod, Paris, 1970.

[Sz1] R. Szőke, Complex structures on tangent bundles of Riemannian manifolds, Math. Ann. 291 (1991), 409-428.

[Sz2] Adapted complex structures and Riemannian homogeneous spaces, Ann. Polon. Math. LXX (1998), 215-220.

[W] R. Wong, Asymptotic approximations of integrals, SIAM, Philadelphia, 2001.

[Wo] N.M.J. Woodhouse, Geometric quantization, 2nd ed., Clarendon Press, Oxford, 1992.

Dept. of Mathematics, Purdue University, West Lafayette, IN 47907, USA

Dept. of Analysis, Institute of Mathematics, Eötvös University, PÁzmány P. sétány 1/C, Budapest 1117, Hungary 